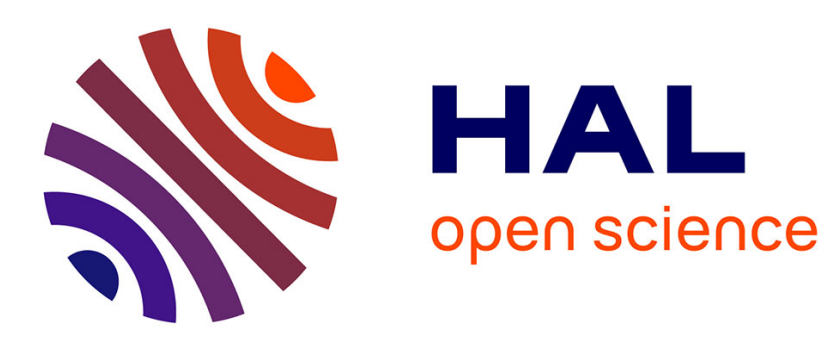

\title{
Parameter Estimation of Superimposed Damped Sinusoids Using Exponential Windows
}

\author{
Muhammad Ali Al-Radhawi, Karim Abed-Meraim
}

\section{To cite this version:}

Muhammad Ali Al-Radhawi, Karim Abed-Meraim. Parameter Estimation of Superimposed Damped Sinusoids Using Exponential Windows. Elsevier Signal Processing Journal, 2014, pp.7. hal-01002078

\section{HAL Id: hal-01002078 https://hal.science/hal-01002078}

Submitted on 13 Jan 2015

HAL is a multi-disciplinary open access archive for the deposit and dissemination of scientific research documents, whether they are published or not. The documents may come from teaching and research institutions in France or abroad, or from public or private research centers.
L'archive ouverte pluridisciplinaire HAL, est destinée au dépôt et à la diffusion de documents scientifiques de niveau recherche, publiés ou non, émanant des établissements d'enseignement et de recherche français ou étrangers, des laboratoires publics ou privés. 


\title{
Parameter Estimation of Superimposed Damped Sinusoids Using Exponential Windows
}

\author{
Muhammad Ali Al-Radhawi ${ }^{\mathrm{a}}$, Karim Abed-Meraim ${ }^{\mathrm{b}}$ \\ ${ }^{a}$ Department of Electrical and Electronic Engineering, Imperial College London, London \\ SW7 2AZ, United Kingdom \\ ${ }^{b}$ Signal and Image Processing Department, Telecom-ParisTech, 75634 Paris Cedex 13, \\ France
}

\begin{abstract}
This paper presents a preprocessing technique based on exponential windowing (EW) for parameter estimation of superimposed exponentially damped sinusoids. It is shown that the EW technique significantly improves the robustness to noise over two other commonly used preprocessing techniques: subspace decomposition and higher order statistics. An ad-hoc but efficient approach for the EW parameter selection is provided and shown to provide close to CRB performance.

Keywords: Exponentially Damped Sinusoids, Colored Noise, Cyclo-stationarity, Complex Exponential Windowing, Cramer-Rao Bound
\end{abstract}

\section{Introduction}

Parameter estimation of superimposed exponentially damped sinusoids from a set of noisy observation data is a problem one frequently encounters

Email addresses: m.rizvi110imperial.ac.uk (Muhammad Ali Al-Radhawi), abed@telecom-paristech.fr (Karim Abed-Meraim) 
in many applications such as nuclear magnetic resonance imaging [1], power systems [2], audio modeling [3], mechanical systems [4], physics [5], chemistry [6] and others. This problem has received considerable attention in the signal processing community in the last decades and is now considered by many to be "solved". Indeed, there are plenty of "solutions" available to this problem. The best known methods include the Prony's method [7, 8], the iterative quadratic maximum likelihood (IQML) method [9, 10], the matrix pencil (MP) method [8], and state space-based method [11]. In addition to the above methods, there are many tools available to preprocess (i.e., clean up) the raw data before any of the above methods is applied. The most popular ones of these tools are subspace decomposition (SD) $[7,8]$ and higher order statistics (HOS) [12, 13].

In the last few years, renewed interest in the sinusoidal estimation problem has been observed either for dedicated applications $[3,1]$ or for adverse situations such as irregular sampling [14], impulsive noise [15] or colored noise [16].

In this work, we consider a colored noise context and we discuss the idea of preprocessing the data with complex exponential windowing. Although there are various ways to modify and improve the Prony's method, the IQML method, or the MP method with a preprocessing step, using SD or HOS is known to be the most effective in reducing the noise effect. However, the SD method, which separates "signal subspace" from "noise subspace", assumes that the noise is either white, or colored with known covariance up to a scalar. The HOS method works well only with Gaussian noise and long data length.

We show that the concept of cyclostationarity (CS) $[17,18]$ or more gener- 
ally exponential windowing $(\mathrm{EW})$ can be easily applied to better preprocess the superimposed exponentially damped sinusoids. The CS or EW methods preserve the signal structure but mitigate the noise effectively as long as it is stationary. Compared to the SD and HOS methods, the CS or EW methods require the least assumption on the noise and hence perform the best. This advantage will be clearly illustrated by simulation results. We propose simple approaches to optimize the EW parameters in such a way the signal power or the post-processing signal to noise ratio (SNR) is maximized. As a benchmark, we derive the Cramér-Rao bound (CRB) expression and use it to illustrate the closeness of the proposed method's performance to the CRB.

The organization of this paper is as follows. In section II, the idea behind using cyclo-stationarity for pre-processing and a generalized exponential windowing method are introduced. Section III is devoted to performance investigations. First, we review the CRB expression for the colored noise case. Then, we evaluate the asymptotic covariance matrix of the pre-processed noise term. The latter is evaluated for white noise and used to express the averaged signal-to-noise ratio (after data pre-processing) that is used for efficient EW parameter selection. In section IV, simulation results are presented demonstrating the superiority of the proposed method. Finally, we state the conclusion. 


\section{Proposed Signal Preprocessing Methodology}

\subsection{Data Model}

The data $y(n)$ under consideration is modeled as follows:

$$
y(n)=x(n)+w(n)=\sum_{m=1}^{L} h_{m} e^{b_{m} n}+w(n), \quad n=0,1, \cdots, N-1
$$

where $h_{m}=a_{m} e^{j \theta_{m}}, b_{m}=\alpha_{m}+j \omega_{m}$ with $\alpha_{m}<0$, and $w(n)$ denotes the stationary noise ${ }^{1} . a_{m}$ and $\theta_{m}$ are respectively the amplitude and the initial phase of the $m^{\text {th }}$ sinusoid; its damping and frequency factors are respectively $\alpha_{m}$ and $\omega_{m}$.

\subsection{Preprocessing Using Cyclo-Stationarity}

Considering the fact that exponentially damped signals have relatively short (effective) length, we apply the CS concept only in the context of second order statistics (SOS) as opposed to HOS. Define the $k^{\text {th }}$ lag $(k \geq 0)$ cyclo-correlation $R^{\beta}(k)$ at the cyclo-frequency $\beta$ as:

$$
R_{\beta}(k):=\sum_{n=0}^{N-k-1} y(n+k) y^{*}(n) e^{j \beta n}
$$

where $^{*}$ denotes the complex conjugate transpose operator. Assuming large sample size ${ }^{2}$, and using (1) in (2) yields

$r_{\beta}(k)=\sum_{m, l=1}^{L} h_{m} h_{l}^{*} e^{k b_{m}} \sum_{n=0}^{N-1-k} e^{\left(b_{m}+b_{l}^{*}+j \beta\right) n}+\mathbb{E} v(k) \approx \sum_{m=1}^{L} A_{\beta}(m) e^{k b_{m}}+\mathbb{E} v(k)$

\footnotetext{
${ }^{1}$ Note that, in contrary to most existing methods, the noise can be colored and nonGaussian. The only assumption is its wide sense stationarity.

${ }^{2}$ More precisely, we assume that $e^{\alpha_{m} N}<<1, \forall m=1, \ldots, L$.
} 
where $r_{\beta}(k)=\mathbb{E} R_{\beta}(k)$, and

$$
v(k)=\sum_{n=0}^{N-1-k} w(n+k) w^{*}(n) e^{j \beta n}, \quad A_{\beta}(m)=\sum_{l=1}^{L} \frac{h_{m} h_{l}^{*}}{1-e^{b_{m}+b_{l}^{*}+j \beta}} .
$$

It is seen from (4) that the cyclo-correlation as function of $k$ also consists of superimposed exponentially damped sinusoids and a noise term. The main advantage, in dealing with cyclo-correlation instead of correlation function as considered in standard sinusoidal estimation techniques $[8,9]$, is that noise contribution is considerably reduced in the former case. In fact, due to the stationarity assumption of the noise process, we have:

$$
\lim _{N \rightarrow \infty} \frac{1}{N} \sum_{n=0}^{N-1-k} w(n+k) w^{*}(n) e^{j \beta n}=0
$$

when

$$
\lim _{N \rightarrow \infty} \frac{1}{N} \sum_{n=0}^{N-1-k} w(n+k) w^{*}(n)=\rho(k)
$$

$\rho(k)$ being the $k^{\text {th }}$ correlation factor of the noise process. Generally, for additive colored noise, the signal to noise ratio (SNR) gain, can be considerable since, for $\rho(k) \neq 0$, we have

$$
\lim _{N \rightarrow \infty}\left|\frac{\sum_{n=0}^{N-1-k} w(n+k) w^{*}(n)}{\sum_{n=0}^{N-1-k} w(n+k) w^{*}(n) e^{j \beta n}}\right|=\infty .
$$

Remark 1. In the case where the data length is relatively small, one can enhance the signal component by applying the integral of the cyclo-correlation over an interval of the cyclo-frequency:

$$
\int_{\beta_{0}}^{\beta_{1}} R_{\beta}(k) d \beta=\sum_{m=1}^{L} B_{\beta}(m) e^{b_{m} k}
$$


where

$$
B_{\beta}(m)=\sum_{l=1}^{L} h_{m} h_{l}^{*}\left[j\left(\log \left(1-e^{b_{m}+b_{l}^{*}+j \beta_{1}}\right)-\log \left(1-e^{b_{m}+b_{l}^{*}+j \beta_{0}}\right)\right)+\beta_{1}-\beta_{0}\right] .
$$

Note that this integration is equivalent to replacing the unit-norm exponential weight $w(n)=e^{j \beta n}$ by the weight coefficient

$$
w^{\prime}(n)=\int_{\beta_{0}}^{\beta_{1}} e^{j \beta n} d \beta=e^{j \frac{\beta_{1}+\beta_{0}}{2} n} \operatorname{sinc}\left(\frac{\beta_{1}-\beta_{0}}{2} n\right)
$$

representing the modulation of a unit-norm exponential with a sinc function (where $\operatorname{sinc}(x)=\sin (x) / x)$. Such integration has also the advantage to provide certain robustness against a 'bad' choice of the cyclo-frequency parameter $\beta$ since we consider all cyclo-frequencies in the range $\left[\beta_{0}, \beta_{1}\right]$. Indeed, one can observe in Fig. 2 (section IV) that a 'bad' selection of parameter $\beta$ might lead to poor estimation performance at low or moderate SNRs.

Remark 2. In the case where the noise is complex circular which implies $\mathbb{E}(w(n) w(n+k))=0$, we also can use the following cyclo-correlation:

$$
R_{\beta}^{\prime}(k) \stackrel{\text { def }}{=} \sum_{n=0}^{N-1-k} y(n+k) y(n) e^{j \beta n}
$$

in which the noise term is mitigated due to both the stationarity and the circularity.

\subsection{Generalized Preprocessing Using Exponential Windows}

A generalization of the previous preprocessing method is the use of a complex cyclo-frequency or a complex exponential window (EW). Therefore, we define the $k^{\text {th }}$ lag exponentially windowed signal $R_{W}(k)$ as:

$$
R_{W}(k):=\sum_{n=0}^{N-1-k} y(n+k) y^{*}(n) W^{n}
$$


where $W$ is a complex windowing parameter satisfying $|W| \leq 1$. We retrieve the previous case when $|W|=1$.

Again by assuming large sample size, the expression of the exponentially windowed signal is:

$$
r_{W}(k)=\sum_{m=1}^{L} A_{W}(m) e^{k b_{m}}+\mathbb{E} v(k)
$$

where

$$
v(k)=\sum_{n=0}^{N-1-k} w(n+k) w^{*}(n) W^{n}, \quad A_{W}(m)=\sum_{l=1}^{L} \frac{h_{m} h_{l}^{*}}{1-W e^{b_{m}+b_{l}^{*}}}
$$

The rational behind using a complex windowing parameter is that the exponentially damped signal has a short effective length determined by the damping factor, and the rest of the data samples are almost noise. Therefore, using an exponential window will reduce accumulated noise in the resulting signal and provide higher weight to data samples with higher signal amplitude. This can lead, as will be observed later in section 4, to non-negligible performance gain. Another reason is that if the signal frequencies were pure sinusoids, then the formula (3) will no longer be valid without a damped window $W$. The damped window $|W|<1$ will guarantee the geometric series to converge always.

\section{Performance Investigation}

\subsection{CRB for the damped sinusoid estimation problem with colored noise}

Consider the data model (1). We assume that $w(n)$ is zero-mean stationary Gaussian circular noise with unknown covariance. Define:

$$
\Theta_{d}=\left[\begin{array}{lllllll}
\alpha_{1} & \alpha_{2} & \ldots & \alpha_{L} & \omega_{1} & \omega_{2} \ldots & \omega_{L}
\end{array}\right]^{T}, \Theta_{n}=\left[\begin{array}{lllllll}
a_{1} & a_{2} & \ldots & a_{L} & \theta_{1} & \theta_{2} \ldots & \theta_{L}
\end{array}\right]^{T}
$$


where $\Theta_{d}$ are the desired parameters and $\Theta_{n}$ are the nuisance parameters ${ }^{3}$. Define $\Theta=\left[\begin{array}{ll}\Theta_{d}^{T} & \Theta_{n}^{T}\end{array}\right]^{T}$ to refer to the desired and nuisance parameters. Assume that the noise follows some parametric model and $\Theta_{w}$ represents the parameters of the noise model. Let $\Omega=\left[\Theta^{T} \Theta_{w}^{T}\right]^{T}$ denotes all model parameters.

The CRB, which is given by the diagonal terms of the Fisher Information Matrix (FIM) inverse [19], is a lower bound ${ }^{4}$ on the variance of the model parameters, i.e., $\operatorname{MSE}(\Omega) \geq \operatorname{CRB}(\Omega)=F_{\Omega}^{-1}$, where $F_{\Omega}$ denotes the FIM for parameter $\Omega$ and MSE stands for mean-squared error.

We will utilize the following lemma to reduce $\mathrm{CRB}$ computation to $F_{\Theta}$ only:

Lemma 3.1 ([20]). The elements of the FIM corresponding to the cross terms of $\Theta$ and $\Theta_{w}$ are zeros.

For a circular Gaussian process, the FIM can be written as [19]:

$\left[F_{\Theta}\right]_{i j}=2 \operatorname{Re}\left\{\frac{\partial X^{*}}{\partial \Theta_{i}} \Gamma^{-1} \frac{\partial X}{\partial \Theta_{j}}\right\}+\operatorname{tr}\left(\Gamma^{-1} \frac{\partial \Gamma}{\partial \Theta_{i}} \Gamma^{-1} \frac{\partial \Gamma}{\partial \Theta_{j}}\right)=2 \operatorname{Re}\left\{\frac{\partial X^{*}}{\partial \Theta_{i}} \Gamma^{-1} \frac{\partial X}{\partial \Theta_{j}}\right\}$

where $X=[x(0) x(1) \ldots x(N-1)]^{T}$ and $\Gamma$ is the noise covariance. Note that the second term in (12) is zero since the noise covariance is assumed to be independent from the damped sinusoids parameters.

\subsection{Parameter Selection}

The EW method has an important parameter $W=e^{d+j \beta}$ to be selected. We use the expressions (10) for that purpose. An intuitive idea consists in

\footnotetext{
${ }^{3}$ This paper focuses only on the frequency and damping parameters estimation.

${ }^{4}$ This lower bound applies for all unbiased estimators.
} 
enhancing the signal-to-noise ratio after pre-processing. In the appendix, we derive the asymptotic covariance of the noise, which turns to have a complicated expression depending on the noise auto-covariance function. We compute instead an explicit expression in the white noise case:

Lemma 3.2. If $w(k)$ was zero-mean circular white Gaussian noise. Then, the EW noise $v(k)$ is asymptotically circular white Gaussian noise with autocovariance function:

$$
\rho(k)=\frac{\sigma^{4}}{1-|W|^{2}} \delta(k)
$$

Thus, we can select the EW parameter by maximizing the averaged postprocessing SNR given by:

$$
\max _{d, \beta}\left(1-\left|W^{2}\right|\right)\left\|A_{W}\right\|^{2}
$$

where $A_{W}=\left[A_{W}(1) \ldots A_{W}(L)\right]^{T}$. Note that the noise covariance is independent of $\beta$. Therefore, for a fixed $d$, the maximization problem is interpreted as:

$$
\max _{\beta}\left\|A_{W}\right\|^{2}
$$

However, if we have unknown noise, we cannot compute the SNR. Nevertheless, it turns out that selecting $\beta$ according to (14) and choosing $d$ as the slowest damping can serve as a good choice for a wide range of noise statistics. Indeed, the latter choice for $d$ is motivated by the fact that the 'effective life duration' of the exponentially damped signal $e^{\alpha n}$ is approximately $N=1 /\left(1-e^{\alpha}\right)$. Hence, it is natural to choose a window size corresponding to the desired signal life duration (i.e. choosing $d$ as the slowest damping factor).

As a result, we provide the following iterative algorithm: 
1. Initialize $d=0$ and $\beta$ randomly ${ }^{5}$.

2. Compute the cyclo-correlation as in (7) or (8).

3. Apply a standard method (e.g MP or IQML) to $r_{W}$ to compute the complex frequencies. Compute the complex amplitude via solving a least square problem.

4. Set $d$ as the slowest nonpositive damping and choose $\beta$ by solving (14).

5. If an end criterion ${ }^{6}$ is reached, then stop. Otherwise, go to step 2.

\section{Simulation}

\subsection{Parameter Selection}

The data we consider here is as in $[12,18]$ :

$$
y(n)=e^{b_{1} n}+e^{b_{2} n}+w(n)
$$

where $b_{1}=-0.1+0.52(2 \pi) i, b_{2}=-0.2+0.42(2 \pi) i$. We assume, at first, zeromean circular white Gaussian noise. We apply the cyclo-correlation (7). For each experiment, the sample size is set to $N=100 . N_{r}=100$ independent Monte-Carlo runs are performed to evaluate the statistics.

Figure 1 , shows the plot of the cost function. The optimal value is $(d, \beta)=$ $(-0.55,0.37)$. Note that $\beta=0.37$ is optimal for every fixed $d$.

Figure 2 shows the sensitivity of the method with respect to $\beta$. It is observed that the maximum of the cost function corresponds to the best

\footnotetext{
${ }^{5}$ If any prior knowledge is available it can be used for initialization, e.g. if it's known that all sinusoids are damped, then $d$ can be initialized to be less than zero.

${ }^{6}$ We observed that 4-6 iterations yield acceptable performance.
} 
performance obtained. Note also that optimization is highly needed since bad a choice of $\beta$ causes bad performance.

Figure 3, shows the result when varying $d$ between the best value suggested by (13) versus slowest damping factor suggested by the proposed algorithm in the case of white Gaussian noise. It can be seen that although $d=-0.55$ results in slightly improved results for mid and high SNRs, our proposed algorithm gives very reasonable performance. The comparison with the case $d=0$ is given next.

\subsection{Performance Comparisons}

To illustrate the performance of the EW preprocessing method, we consider the MP algorithm (which uses implicitly the SD technique) [8]. By combining the MP method with HOS [12], we have a method to be called HOS-MP method. By applying the MP method to the exponentially window signal, and using the iterative algorithm for $\beta$, we have the EW-MP method. We use CS-MP to refer to the EW-MP with $d=0$. In the sequel, we compare the MP, HOS-MP, EW-MP and CS-MP methods.

The data we consider is same as previous subsection. The performance is

measured by the mean-square error (MSE) defined as MSE $=\sqrt{\frac{1}{N_{r}} \sum_{r=1}^{N_{r}}\left\|\hat{b}_{r}-b\right\|^{2}}$, where $b$ denotes the parameter vector, i.e., $b=\left[b_{1}, b_{2}\right]^{T}$ and $\hat{b}_{r}$ is the estimate of $b$ at the $r$-th run. For the EW-MP and CS-MP methods, the iterative algorithm was initialized with $(d, \beta)=(0,0)$, and only four iterations were performed. We set the processing window size for all MP methods to 15 (see [8] for more details).

The following cases are considered:

Case 1: White Gaussian circular noise: Figure 4 shows the MSE versus 
SNR. The EW-MP method performs the best for low SNRs even in this case where the noise assumptions required by all methods are satisfied. For medium and high SNR, all methods perform closely.

Case 2: Colored Gaussian noise: The colored noise is generated by the $\operatorname{AR}(a)$ filter

$$
w(n)=a w(n-1)+\text { white Gaussian noise }
$$

where the white Gaussian noise has the power $\sigma^{2}$ (SNR is defined as $-10 \log _{10} \sigma^{2}$ ). Figure 5 shows the MSE versus SNR for $a=0.9$. In this case, the EWMP method performs significantly better for low and medium SNRs. The HOS-MP seems to be highly affected by the small sample size. Note that EW-MP has better performance than CS-MP which illustrates the utility of the damped exponential windowing.

Figure 6 shows the MSE performance between the three methods versus the value of $a$ with $\mathrm{SNR}=20 \mathrm{~dB}$. Clearly, the EW preprocessing increases the robustness of MP against AR noise.

Case 3: White non-Gaussian noise: The noise is complex with real and imaginary parts uniformly distributed over $[-\sigma / \sqrt{2}, \sigma / \sqrt{2}]$. Figure 7 , shows also the superiority of EW-MP method.

Case 4: Colored non-Gaussian noise: In this case, the colored noise is generated filtering the white uniformly distributed by a $\operatorname{AR}(a)$ filter, where $a=0.9$. As shown in Figure 8, both the MP and HOS-MP methods fail to compete with the EW-MP method because the noise assumptions required by the former two are not satisfied. 


\section{Conclusion}

In this paper, we discussed a sinusoids estimation method that works acceptably with wide range of stationary noise. The idea is based on computing the cyclo-correlation (or a generalized exponential window) of the signal. We provided a simple algorithm for EW parameter selection and performance optimization. We showed through simulations that it has a superior performance to other methods especially in the case of non-Gaussian and/or colored noise. Comparisons with the CRB showed that it can give close to CRB performance at moderate and high SNRs.

\section{Appendix A. Derivation of The Asymptotic Covariance of Expo- nentially Windowed Noise}

Theorem Appendix A.1. In (1), assume that $w(k)$ is a stationary zeromean circular Gaussian noise with autocorrelation function $\rho(k)$. Then the covariance of $v(k)$ is given by:

$$
C_{v}(k, \ell)=C_{v}(s)=\sum_{m=0}^{\infty} \sum_{n=0}^{\infty} W^{n} W^{* m} \rho(n-m+s) \rho^{*}(n-m), s=k-\ell
$$

Proof. From (8), we have

$$
v(k)=\sum_{n=0}^{\infty} w(n+k) w^{*}(n) W^{n}
$$

First, we compute the mean of $v(k)$. Using (A.2) we have:

$\mu_{k}=E[v(k)]=\sum_{n=0}^{\infty} E\left[w(n+k) w^{*}(n)\right] W^{n}=\frac{\rho(k)}{1-W}=\rho(k) L, \quad L=\frac{1}{1-W}$. 
Therefore,

$$
C_{v}(k, \ell)=E\left[v(k) v^{*}(\ell)\right]-\mu_{k} \mu_{\ell}^{*}=E\left[v(k) v^{*}(\ell)\right]-|L|^{2} \rho(k) \rho^{*}(\ell)
$$

Using noise gaussianity and circularity, we compute the first term as:

$$
\begin{aligned}
E\left[v(k) v^{*}(\ell)\right] & =E\left[\sum_{m, n} W^{n} W^{* m} w(n+k) w^{*}(n) w^{*}(m+\ell) w(m)\right] \\
& =\sum_{m, n} W^{n} W^{* m} E\left[w(n+k) w^{*}(n) w^{*}(m+\ell) w(m)\right] \\
& =\sum_{m, n} W^{n} W^{* m}\left(E\left[w(n+k) w^{*}(n)\right] E\left[w^{*}(m+\ell) w(m)\right]+E\left[w(n+k) w^{*}(m+\ell)\right]\right. \\
& =\sum_{m, n} W^{n} W^{* m}\left(\rho(k) \rho^{*}(\ell)+\rho(n-m+k-\ell) \rho^{*}(n-m)\right) \\
& =\sum_{m, n} W^{n} W^{* m} \rho(k) \rho^{*}(\ell)+\sum_{m, n} W^{n} W^{* m} \rho(n-m+k-\ell) \rho^{*}(n-m) \\
& =|L|^{2} \rho(k) \rho^{*}(\ell)+\sum_{m, n} W^{n} W^{* m} \rho(n-m+k-\ell) \rho^{*}(n-m)
\end{aligned}
$$

and the result follows.

We can proof Lemma 3.2 now:

Proof of Lemma 3.2. Set $\rho(k)=\delta(k)$. If $s \neq 0$, we have $C_{v}(s)=0$. If $s=0$ then:

$$
\begin{aligned}
C_{v}(s) & =\sigma^{4} \sum_{m=0}^{\infty} \sum_{n=0}^{\infty} W^{n} W^{* m} \delta(n-m+s) \delta^{*}(n-m) \\
& =\sigma^{4} \sum_{n=0}^{\infty}|W|^{2 n}=\frac{\sigma^{4}}{1-|W|^{2}}
\end{aligned}
$$




\section{References}

[1] D. Rubtsov, J. Griffin, Time-domain Bayesian detection and estimation of noisy damped sinusoidal signals applied to NMR spectroscopy, Journal of Magnetic Resonance 188 (2) (2007) 367-379.

[2] L. Lovisolo, M. Tcheou, E. da Silva, M. Rodrigues, P. Diniz, Modeling of electric disturbance signals using damped sinusoids via atomic decompositions and its applications, EURASIP Journal on Applied Signal Processing 2007 (1) (2007) 168.

[3] R. Boyer, K. Abed-Meraim, Audio modeling based on delayed sinusoids, IEEE Transactions on Speech and Audio Processing 12 (2) (2004) 110120.

[4] S. Sarma, An adaptive nonlinear filter for online parameter estimation of undamped and damped sinusoids, Mechanical Systems and Signal Processing 21 (2) (2007) 1026-1040.

[5] E. Berti, V. Cardoso, J. Gonzalez, U. Sperhake, Mining information from binary black hole mergers: a comparison of estimation methods for complex exponentials in noise, Physical Review D 75 (12) (2007) 124017.

[6] J. Papy, L. De Lathauwer, S. Van Huffel, Exponential data fitting using multilinear algebra: The decimative case, Journal of Chemometrics 23 (2009) 341-351.

[7] R. Kumaresan, D. W. Tufts, Estimating the parameters of exponentially 
damped sinusoids and pole-zero modeling in noise, IEEE Transactions on Acoustics Speech and Signal Processing 30 (1982) 833-840.

[8] Y. Hua, T. Sarkar, Matrix pencil method for estimating parameters of exponentially damped/undamped sinusoids in noise, IEEE Transactions on Acoustics Speech and Signal Processing 38 (5) (1990) 814-824.

[9] Y. Bresler, A. Macovski, Exact maximum likelihood parameter estimation of superimposed exponential signals in noise, IEEE Transactions on Acoustics, Speech and Signal Processing 34 (5) (1986) 1081-1089.

[10] Y. Hua, The most efficient implementation of the IQML algorithm, IEEE Transactions on Signal Processing 42 (8) (1994) 2203-2204.

[11] J. Lu, L. Brown, Internal model principle-based control of exponentially damped sinusoids, International Journal of Adaptive Control and Signal Processing, In press.

[12] C. Papadopoulos, C. Nikias, Parameter estimation of exponentially damped sinusoids using higherorder statistics, IEEE Transactions on Acoustics, Speech and Signal Processing 38 (8) (1990) 1424-1436.

[13] D. Ruiz, M. Carrion, A. Gallego, A. Medouri, Parameter estimation of exponentially damped sinusoids using a higher order correlation-based approach, IEEE Transactions on Signal Processing 43 (11) (1995) 26652677.

[14] E. Gudmundson, J. Ling, P. Stoica, J. Li, A. Jakobsson, Spectral Estimation of Damped Sinusoids in the Case of Irregularly Sampled Data, in: 
International Symposium on Signals, Circuits and Systems (ISSCS09), 2009 .

[15] M. Altınkaya, H. Deliç, B. Sankur, E. Anarım, Subspace-based frequency estimation of sinusoidal signals in alpha-stable noise, Signal Processing 82 (12) (2002) 1807-1827.

[16] P. Stoica, A. Nordsjö, Subspace-based frequency estimation in the presence of moving-average noise using decimation, Signal Processing 63 (3) (1997) 211-220.

[17] W. Gardner, Exploitation of spectral redundancy in cyclostationary signals, IEEE Signal Processing Magazine 8 (2) (1991) 14-36.

[18] K. Abed-Meraim, A. Belouchrani, A. Mansour, Y. Hua, Parameter estimation of exponentially damped sinusoids using second order statistics, in: Proc. of Europ. Signal Processing Conf. (EUSIPCO), 1998.

[19] P. Stoica, R. Moses, Spectral analysis of signals, Pearson Prentice Hall, 2005.

[20] J. Francos, B. Friedlander, Bounds for estimation of complex exponentials in unknown colored noise, IEEE Transactions on Signal Processing 43 (9) (1995) 2176-2185. 


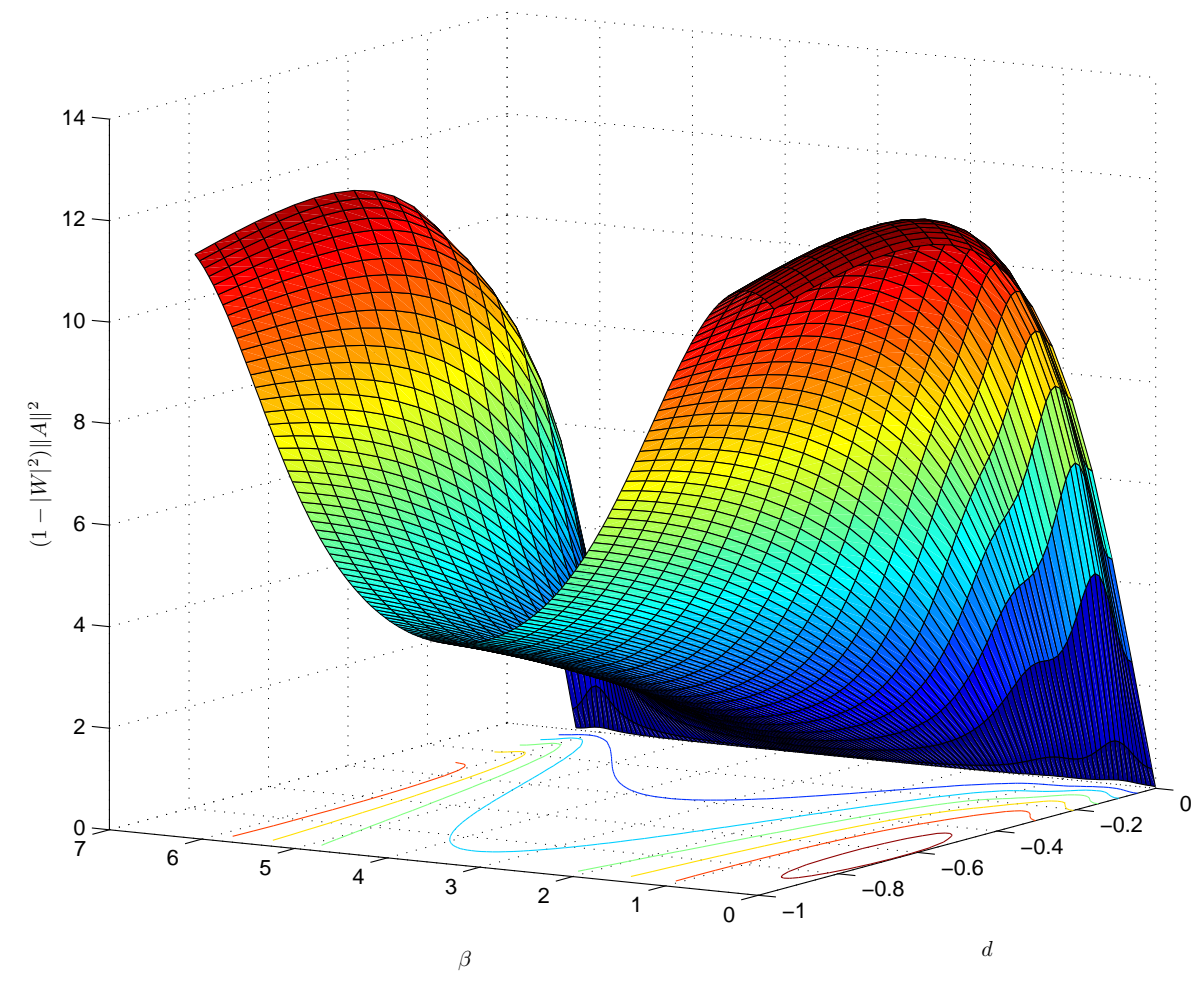

Figure 1: Plot of the cost function in (13). 


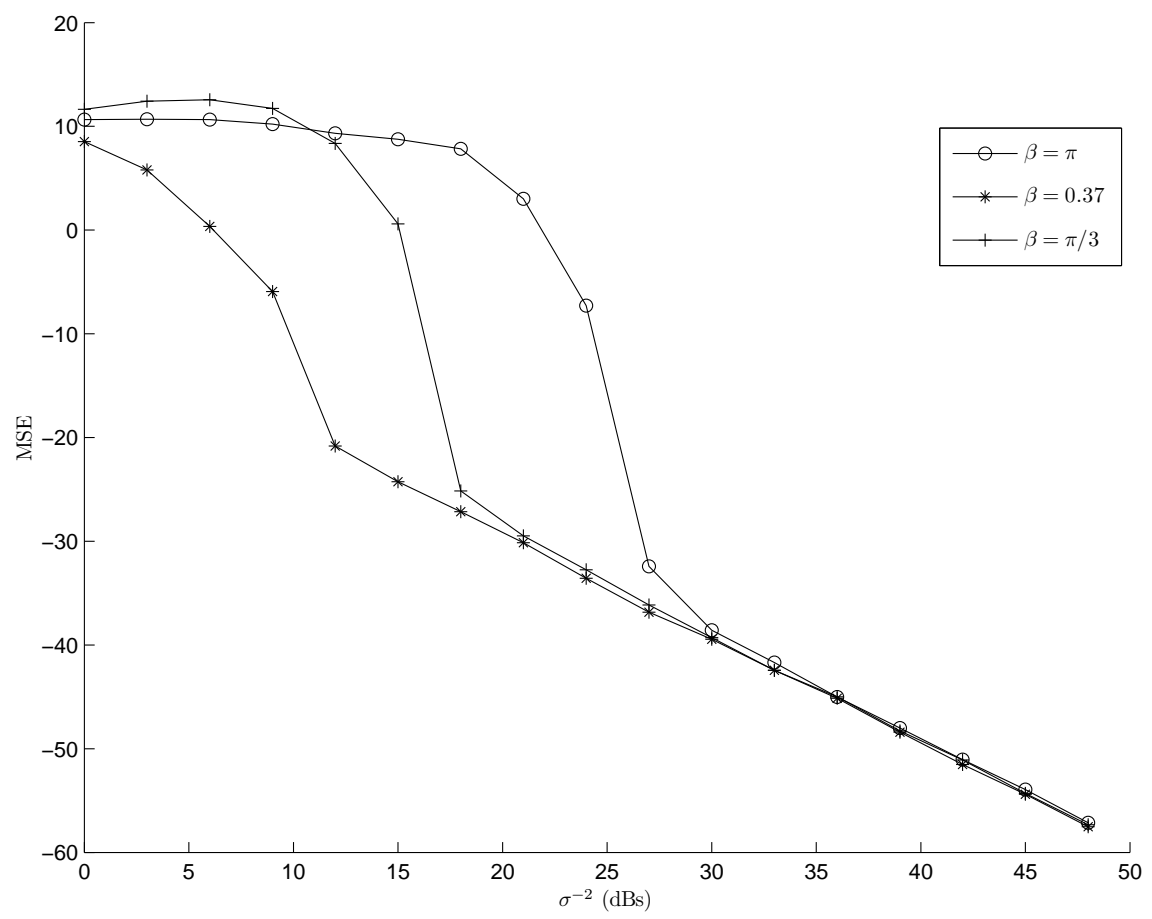

Figure 2: The MSE curves with different values of $\beta$ with fixed $d=-0.1$ in the case of white Gaussian noise. 


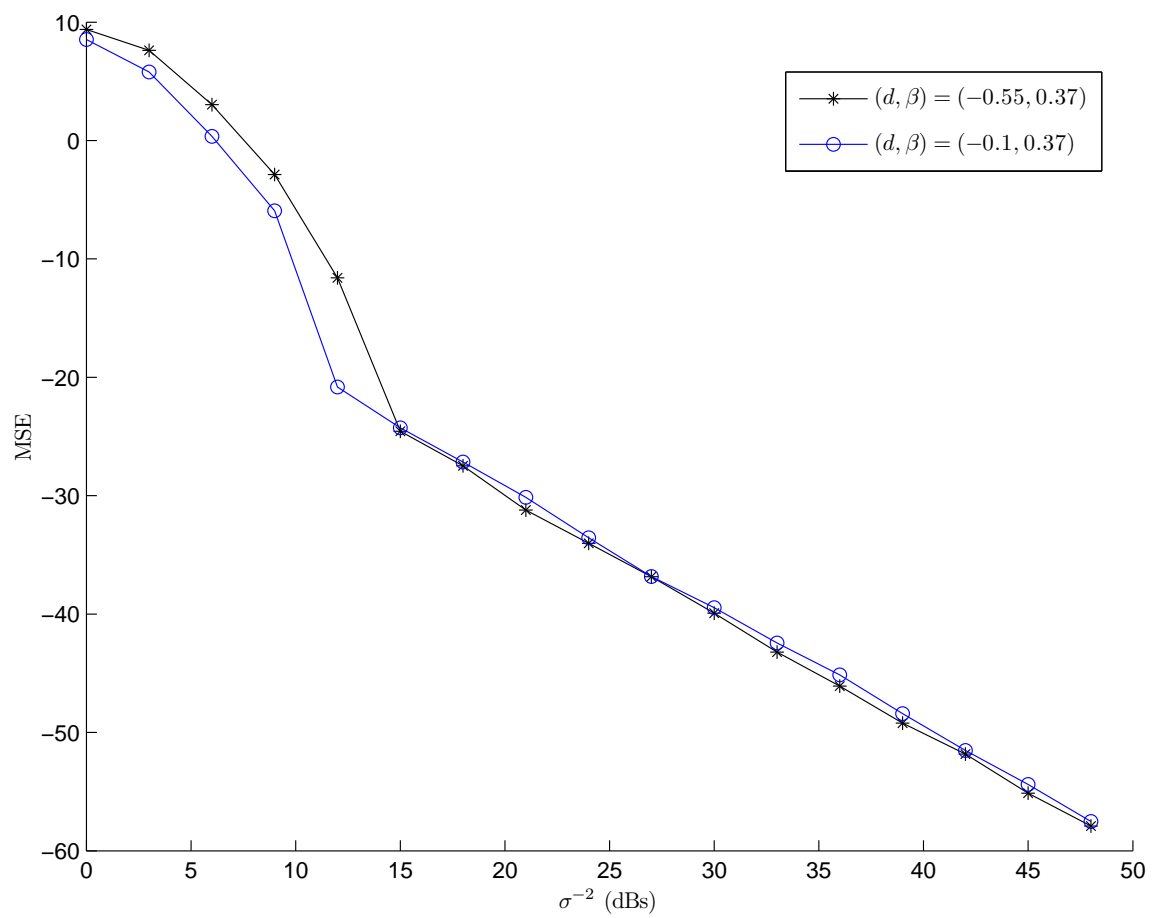

Figure 3: MSE curves comparing the performance between the optimization (13) and the proposed algorithm in the case of white Gaussian noise. 


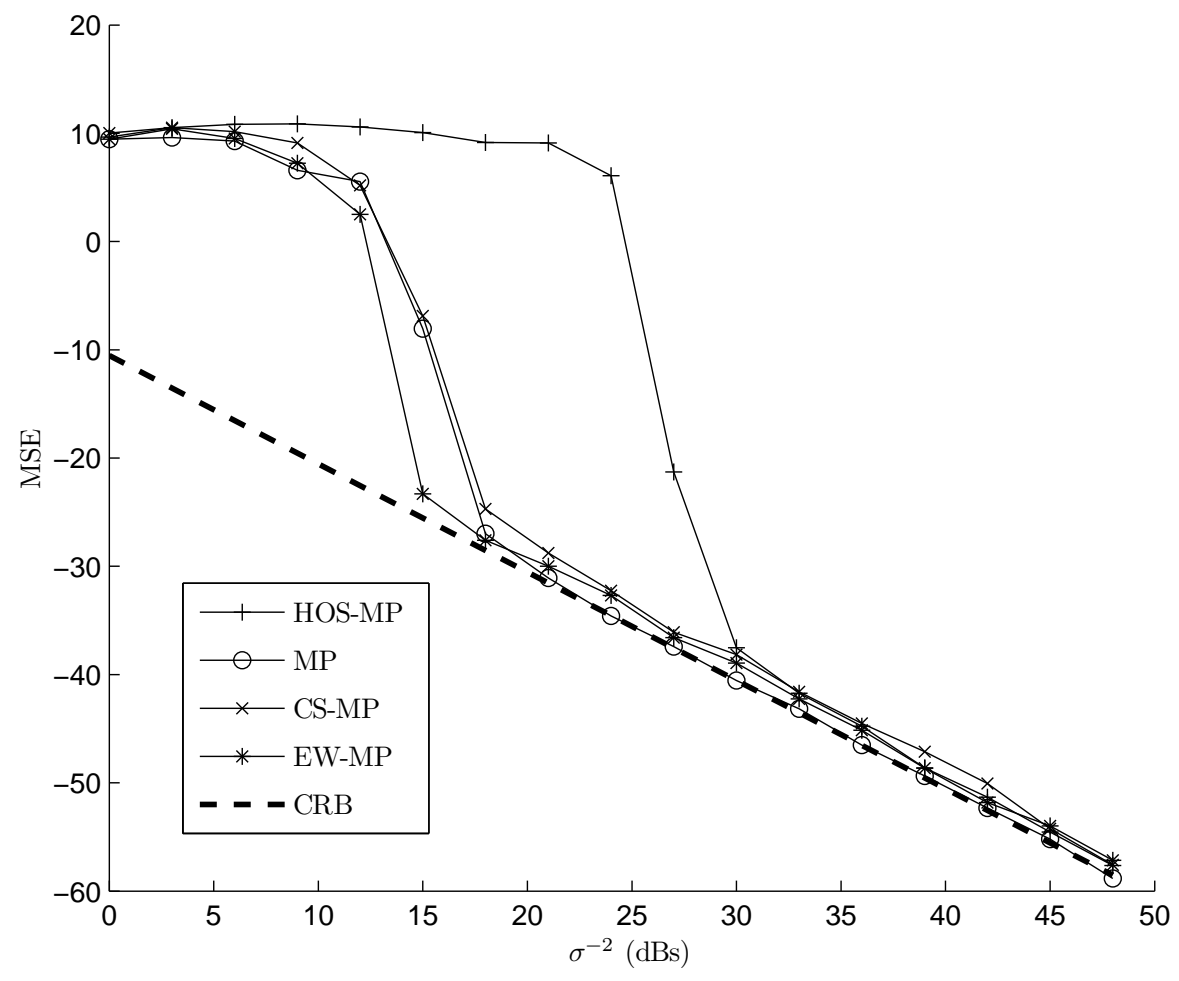

Figure 4: White Gaussian circular noise case. 


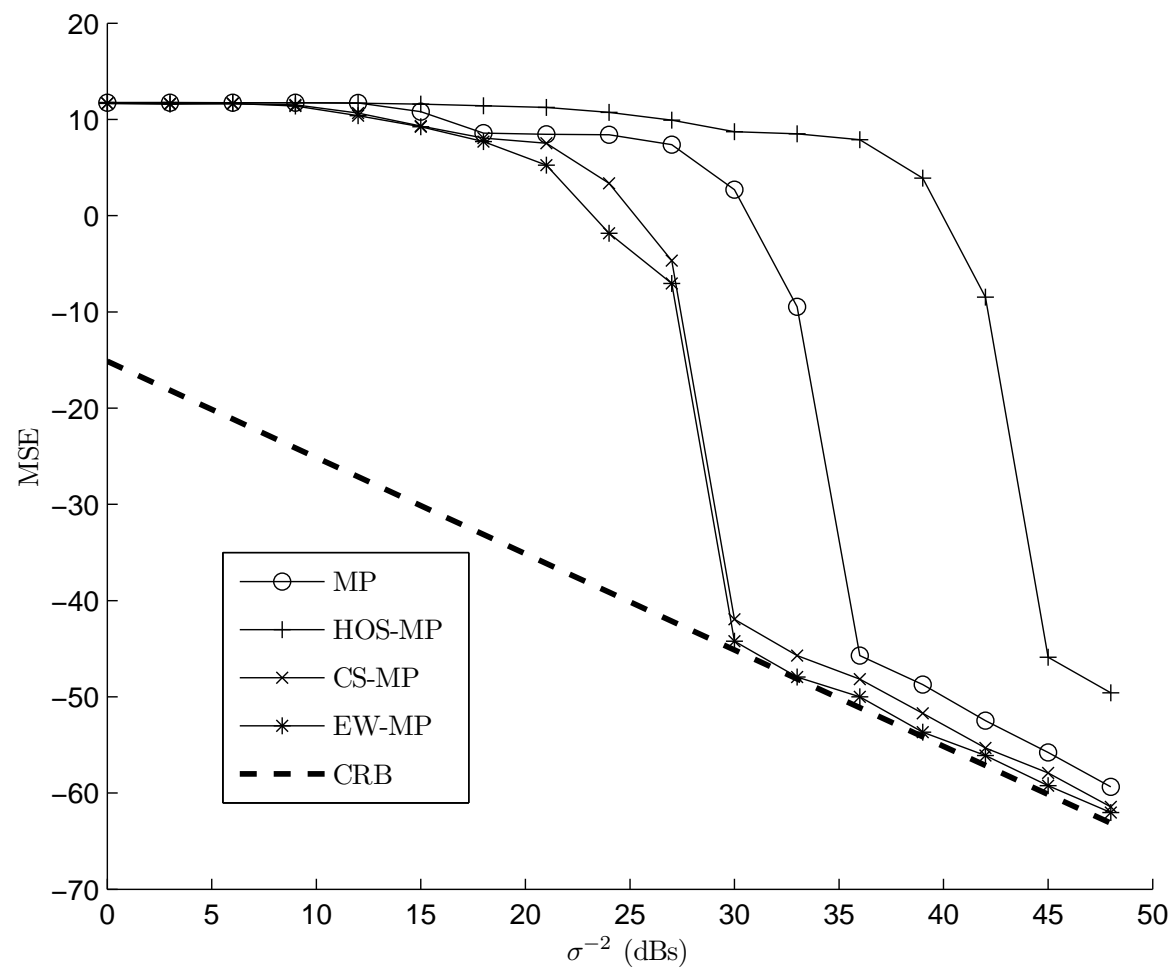

Figure 5: AR(0.9) Gaussian circular noise case. 


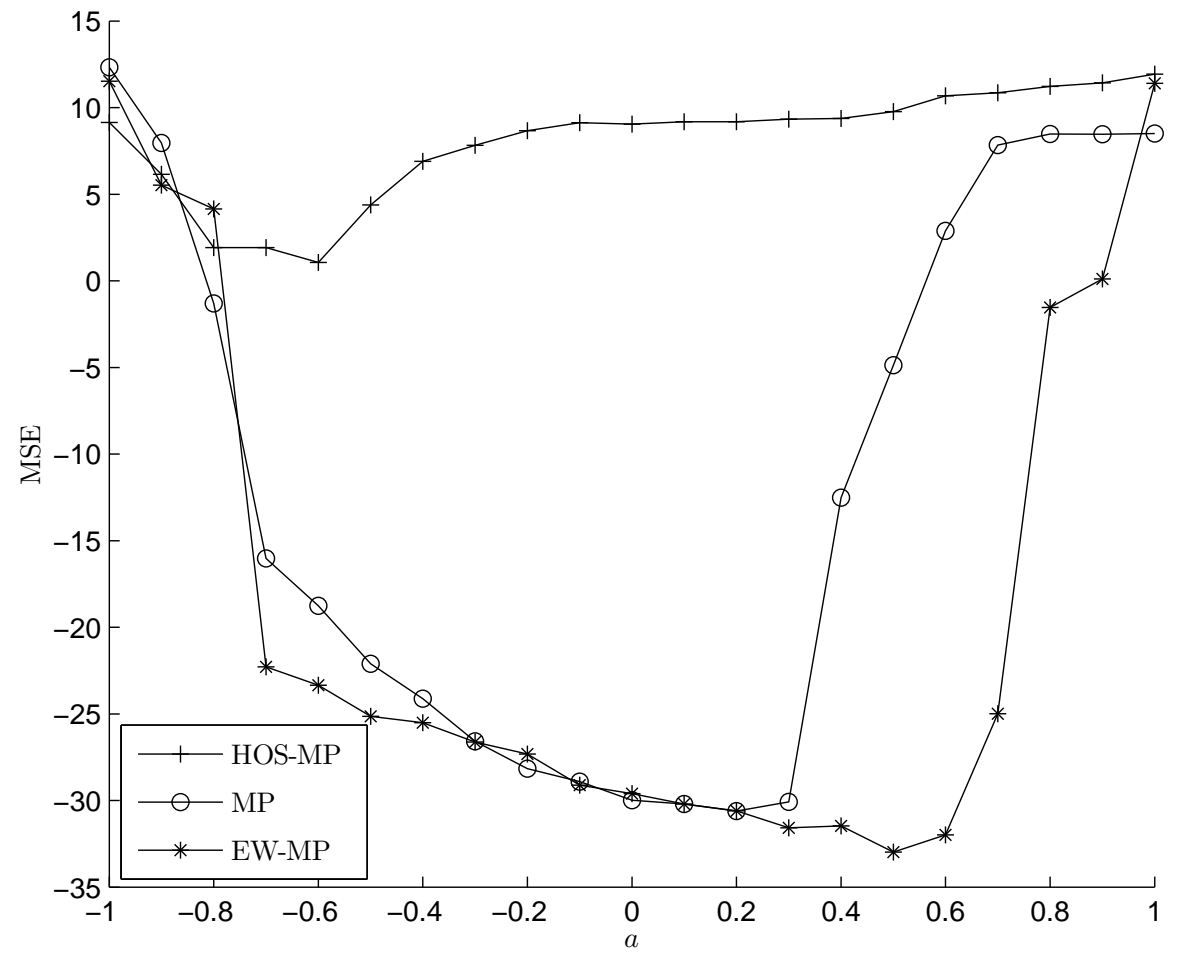

Figure 6: The MSE with AR colored Gaussian noise versus $a$ for $\mathrm{SNR}=20 \mathrm{~dB}$. 


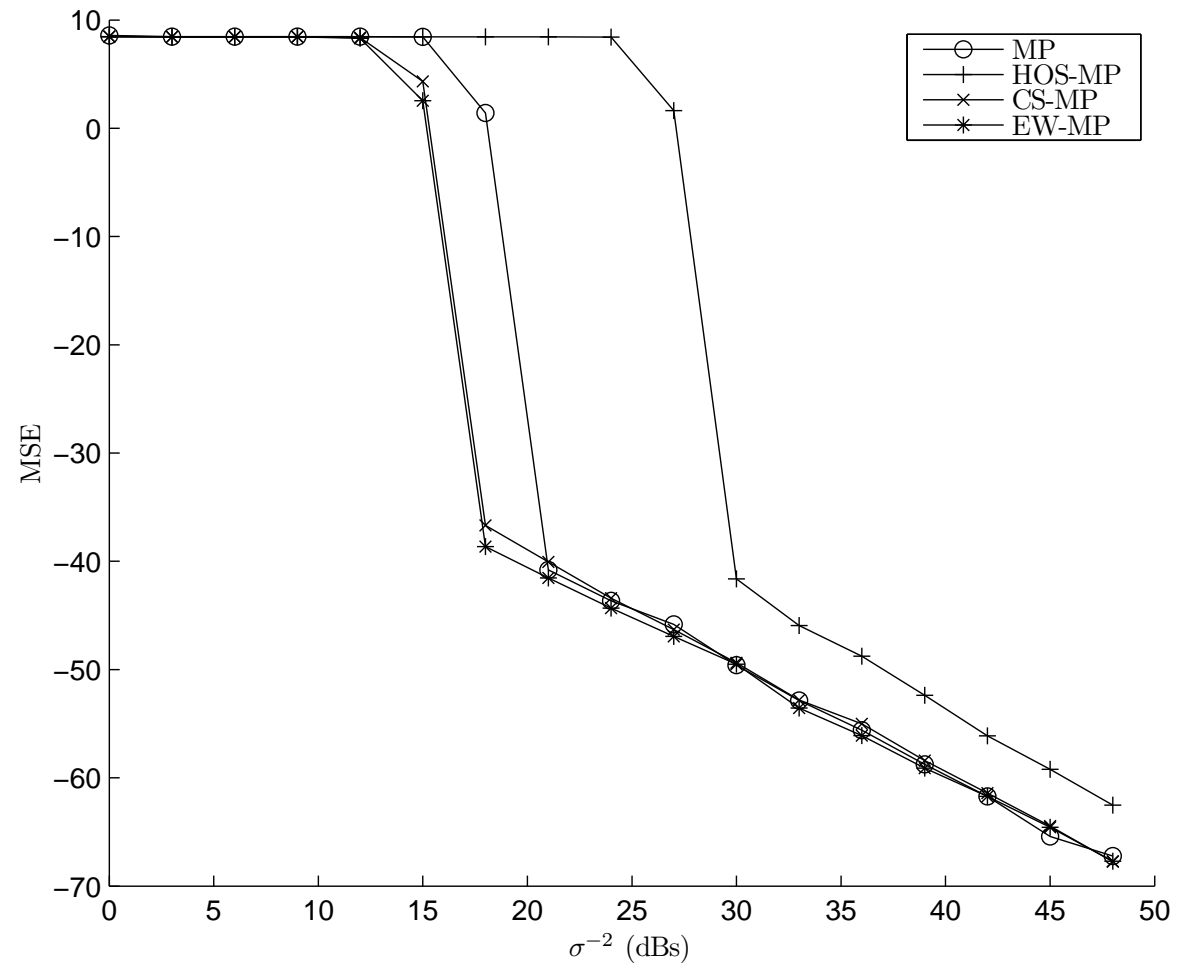

Figure 7: The case of white uniformly distributed noise. 


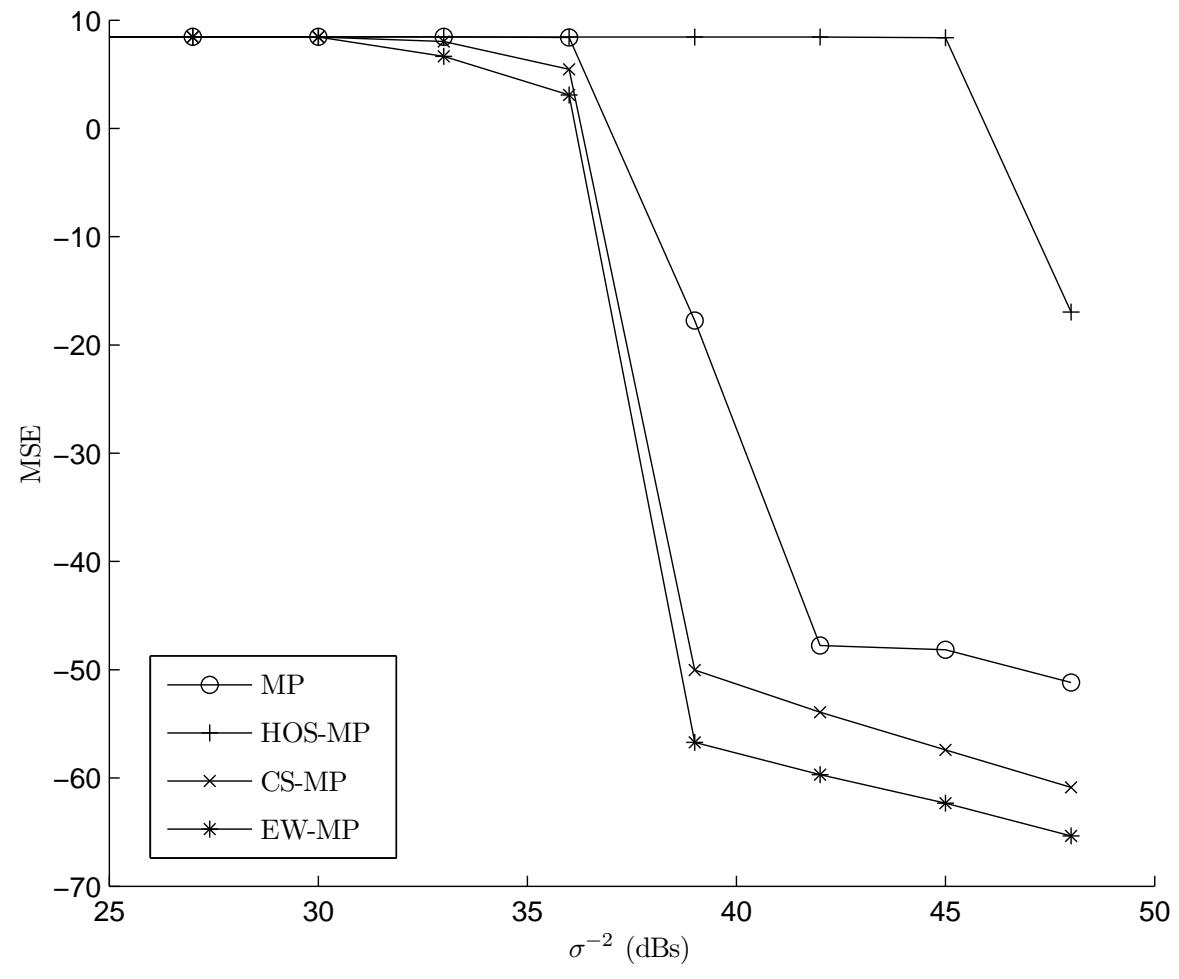

Figure 8: The case of $\mathrm{AR}(0.9)$ uniformly distributed noise. 